\title{
Exendin-4 promotes osteogenic differentiation of adipose-derived stem cells and facilitates bone repair
}

\author{
BANGLIAN DENG ${ }^{1,2}$, WENZHONG ZHU ${ }^{3}$, YANSHENG DUAN ${ }^{1,2}$, YUQIAN HU $^{4}$, \\ XUEFENG CHEN $^{5}$, SHUANG SONG ${ }^{6}$, ZIAN YI $^{7}$ and YINGLIANG SONG ${ }^{1}$
}

\begin{abstract}
${ }^{1}$ State Key Laboratory of Military Stomatology and National Clinical Research Center for Oral Diseases and Shaanxi Key Laboratory of Stomatology, Department of Oral Implantation, School of Stomatology, The Fourth Military Medical University, Xi'an, Shaanxi 710032; ${ }^{2}$ Department of Dentistry, The People's Hospital of Ankang, Ankang, Shaanxi 725000; ${ }^{3}$ Department of Stomatology, Shaanxi Province Geriatric Hospital, Xi'an, Shaanxi 710005; ${ }^{4}$ Department of Stomatology, The Faculty of Medicine, Eastern University of Liaoning, Shenyang, Liaoning 110000; ${ }^{5}$ Xuefeng Dental Care Huaian, Huaian, Jiangsu 223000; ${ }^{6}$ Health Science Center, Peking University, Beijing 100000; ${ }^{7}$ Department of Stomatology, Kunming Medical University, Kunming, Yunnan 650500, P.R. China
\end{abstract}

Received June 3, 2019; Accepted September 10, 2019

DOI: $10.3892 / \mathrm{mmr} .2019 .10764$

\begin{abstract}
Inflammation-related bone defects pose a heavy burden on patients and orthopedic surgeons. Although stem-cell-based bone repair has developed rapidly, it is of great significance to characterize bio-active molecules that facilitate bone regeneration. It is reported that a glucagon-like peptide 1 receptor agonist, exendin-4, promoted bone regeneration mediated by the transplantation of adipose-derived stem cells in a metaphyseal defect mouse model of femur injury. However, the underlying mechanism is unclear. Bone imaging, immunohistochemistry real-time PCR and western blot analysis were used in the present study, and the results revealed that exendin- 4 increased the transcription of the osteogenic differentiation-related genes and induced osteogenic differentiation in situ. Furthermore, the present data obtained from sorted adipose-derived stem cells revealed that exendin- 4 promoted osteogenic differentiation and inhibited adipogenic differentiation in vitro. These findings indicated that exendin- 4 facilitates osteogenic differentiation
\end{abstract}

Correspondence to: Dr Yingliang Song, State Key Laboratory of Military Stomatology and National Clinical Research Center for Oral Diseases and Shaanxi Key Laboratory of Stomatology, Department of Oral Implantation, School of Stomatology, The Fourth Military Medical University, 15 Changle West Road, Xi'an, Shaanxi 710032, P.R. China

E-mail: songyingliang@163.com

Abbreviations: ADSC, adipose-derived stem cells; BMSCs, bone marrow stromal stem cells; PKA, protein kinase A; GLP-1, glucagon-like peptide 1; bFGF, basic fibroblast growth factor; OPG, osteoprotegerin; ALP, alkaline phosphatase

Key words: exendin-4, bone defect, ADSC, osteoblast differentiation, bone repair of transplanted adipose-derived stem cells for bone repair and illuminated clinical prospects of both adipose-derived stem cells and exendin- 4 in stem-cell-based bone defect repair.

\section{Introduction}

Promoting bone defect repair following trauma, resection, or systemic inflammation is a great challenge for physicians. Both autografts and allografts for large-scale bone repair require invasive surgeries, and the recovery varies from one patient to another. With the rapid development of tissue engineering, adult stem cells have been revealed to accelerate bone repair and improve outcomes (1-5). Adipose-derived stem cells (ADSCs) possess the potential to differentiate into multiple cell lineages including osteoblasts (6,7). Notably, since ADSCs can be easily accessed from fatty tissue and the procedure causes minimal discomfort compared to procedures used to obtain other types of adult stem cells, ADSCs are emerging as a competitive adult stem cell source for bone defect repair (7-9). Furthermore, surface markers of ADSCs have been well-characterized, providing standardized protocols for their isolation and characterization (10). More importantly, ADSCs are capable of secreting trophic factors that facilitate bone repair in situ $(11,12)$. However, it is critical to induce ADSCs with external cues to initiate osteogenic differentiation and to simultaneously inhibit non-osteogenic routes, such as adipogenic differentiation.

Osteogenic differentiation of ADSCs is elaborately regulated by genetic networks and external stimuli. Bone morphogenetic protein (BMP) signaling, extracellular signal-regulated kinase (ERK) signaling, Wnt signaling, and Notch signaling have all been revealed to be involved in the regulatory network of ADSC differentiation (13-16). Recently, several bio-active molecules were revealed to play roles in regulating ADSC differentiation. A histone deacetylase inhibitor was revealed to have a pro-osteogenic effect on rat ADSCs by inducing histone hyper-acetylation at the promoter 
region of Runx2 (17), a well-characterized factor that controls the osteogenic differentiation of ADSCs (18-20). Notably, melatonin and vitamin $\mathrm{D}$ were demonstrated to inhibit adipogenic differentiation of human ADSCs (21), further revealing that the differentiation of ADSCs during clinical bone repair or other clinical events can potentially be modulated by bio-active molecules.

Exendin-4 is a biologically active peptide with a length of 39 amino acids originally isolated from the venom of Heloderma suspectum, the Gila monster lizard. It was initially revealed to promote amylase release from the pancreatic acini of both guinea pigs and rats. Exendin-4 belongs to the glucagon superfamily of peptide hormones whose function can be antagonized by the exendin receptor inhibitor exendin (9-39) (22-24). The synthetic version of exendin-4, exenatide, was approved in 2005 for treatment of type 2 diabetes by improving glycemic control $(25,26)$. Notably, the murine receptor for exendin-4 is essential for the control of bone resorption, as evidenced by GLP-1 receptor knockout mice (27). Moreover, exendin-4 also exhibited a protective role in osteopenia by promoting bone formation and inhibiting bone resorption $(28,29)$. Recently, accumulating evidence has demonstrated different roles of exendin-4 in osteogenic differentiation. In MC3T3-E1 cells, treatment with exendin-4 promoted proliferation and differentiation by upregulating the phosphorylation level of the MAPK (mitogen-activated protein kinase) signaling kinases ERK1/2, p38, and JNK (30), suggesting a role of exendin- 4 in regulating osteogenic differentiation. Furthermore, exendin-4 treatment could not only protect bone marrow stromal stem cells (BMSCs) from mitogen-deprivation-induced apoptosis (31) but also promoted osteogenic differentiation and inhibited adipogenic differentiation of BMSCs by regulating PKA (Protein Kinase A)/ $\beta$-catenin signaling (32). Notably, a recent study revealed that exendin-4 inhibited lipopolysaccharide-induced osteoclast formation and bone resorption via inhibition of TNF- $\alpha$ expression in macrophages (33). Collectively, the data indicate that exendin- 4 holds great promise for promoting osteogenic differentiation in adult stem cells and facilitating bone repair.

In the present study, the role that exendin- 4 plays in ADSC-mediated bone defect repair in vivo was examined by establishing a corresponding mouse bone defect model. In addition, osteogenic and adipogenic differentiation of ADSCs both in vivo and in vitro under exendin- 4 supplementation were investigated and it was revealed that exendin- 4 promoted the osteogenic differentiation of ADSCs. Moreover, the present results also indicated that exendin-4 treatment increased the mRNA and protein levels of genes related to osteogenic differentiation. The present data further indicated the clinical potential of exendin-4 in improving the osteogenic differentiation of ADSCs, which holds great promise for bone defect repair based on tissue engineering.

\section{Materials and methods}

ADSC isolation. All animal experiments were performed in accordance with the guidelines of the NIH (Publication no. 85e23 Rev. 1985) and were approved by the Animal Care and Use Committee of The Fourth Military Medical University. C57 mice were purchased from the Animal Center of The Fourth Military Medical University, and housed in an environmentally controlled room $\left(20-25^{\circ} \mathrm{C}\right)$ with a $12 \mathrm{~h}$ light/dark cycle and free access to food and water. Inguinal fat pads from C57 black/DBA male mice (3 months) were finely minced and digested with $0.2 \%$ collagenase type $\mathrm{I}$ in a $37^{\circ} \mathrm{C}$ shaking incubator for $45 \mathrm{~min}$. The digested tissue was filtered through a sterile $100-\mu \mathrm{m}$ nylon mesh, centrifuged $(300 \mathrm{x} \mathrm{g}$ at $37^{\circ} \mathrm{C}$ for $\left.8 \mathrm{~min}\right)$, resuspended, and cultured in regular growth medium, consisting of $\alpha$-minimum essential medium ( $\alpha$-MEM; HyClone; GE Healthcare Life Sciences), 10\% fetal bovine serum (FBS; Gibco BRL; Thermo Fisher Scientific, Inc.), and penicillin/streptomycin (Sigma-Aldrich; Merck $\mathrm{KGaA}$ ). Cell cultures were maintained at $37^{\circ} \mathrm{C}$ in a humidified incubator with $5 \% \mathrm{CO}_{2}$. Passage 3 cells were used for identifying ADSC phenotypes and for the following experiments.

Bone defect model. The mouse model of metaphyseal defect of the femur was established as previously described (34). Briefly, C57 black/DBA male mice (3 months, weight 23-27 g, $\mathrm{n}=24$ ) were used to establish the bone defect model under anesthesia via an intraperitoneal injection of $300 \mathrm{mg} / \mathrm{kg}$ avertin (Sigma-Aldrich; Merck KGaA) $(35,36)$. Anesthetic depth was confirmed by dilated pupils, loss of pain, loss of palpebral reflex and corneal reflexes present. The side effect of avertin, intestinal ileus, was not observed in these experiments. After making a $10-\mathrm{mm}$ incision, a blunt $0.9 \mathrm{~mm}$ drill was used to drill through the anterolateral cortical bone into the metaphyseal cancellous bone to generate a round defect at the supracondylar region of the right femur. The right hind limbs of three-month-old wild-type animals were used as the control group. Hydrogels combined with $3 \times 10^{5}$ ADSCs were injected into the defective site after the operation. Exendin-4 (Sigma-Aldrich; Merck KGaA) was administered intraperitoneally at $4.2 \mu \mathrm{g} / \mathrm{kg} /$ day, as previously described (37). The mice were sacrificed by cervical dislocation at day 60 following the surgery.

Bone imaging. Femurs were removed from sacrificed mice for micro-computed tomography $(\mu \mathrm{CT})$ analysis and biomechanical testing. The samples were scanned using the Explore Locus SPPre-clinical Specimen Micro-CT (GE Healthcare Life Sciences), and the images were reconstructed to an isotropic voxel size of $12 \mu \mathrm{m}$. All three-dimensional (3D) image manipulations and analyses were performed by the system software (MicroView, v.2.1; GE Healthcare Life Sciences).

Histomorphometry and histology. Mice were injected intraperitoneally with $25 \mathrm{mg} / \mathrm{kg}$ tetracycline and $5 \mathrm{mg} / \mathrm{kg}$ calcein (both from Sigma-Aldrich; Merck KGaA) 10 days later for histomorphometric analysis. For hematoxylin and eosin (H\&E) staining, paraffin sections were prepared. Four-micrometer-thick sections were stained with $\mathrm{H} \& \mathrm{E}$ (Sigma; Merck KGaA; $37^{\circ} \mathrm{C}, 30 \mathrm{~min}$ ) to count the adipocytes.

Immunohistochemistry. Briefly, serial paraffin sections $(5 \mu \mathrm{m})$ were prepared, deparaffinized, and incubated in $3 \% \mathrm{H}_{2} \mathrm{O}_{2}$ to block native peroxidases, followed by incubation at room temperature for $30 \mathrm{~min}$ with non-immune animal serum. Immune-histochemical reactions using antibodies reactive against COL-1 (1:500, cat. no. ab109025; Abcam), CD29 
(1:1,000, cat. no. 102225), or Sca-1 (1:1,000, cat. no. 122504; both from BioLegend, Inc.) were conducted at $4^{\circ} \mathrm{C}$ overnight, followed by horseradish peroxidase (HRP)-conjugated secondary antibody (1:1,000, cat. no. ab6721; Abcam) treatment for $30 \mathrm{~min}$, and washing with phosphate-buffered saline. Antibody binding was detected using an SP immunohistochemistry kit (Abcam) and DAB Horseradish Peroxidase Color Development kit (Thermo Fisher Scientific, Inc.).

Reverse transcription-quantitative $(R T-q) P C R$. For each sample, $500 \mathrm{ng}$ of total RNA was reverse-transcribed using the QuantiTect reverse transcription kit (Qiagen $\mathrm{GmbH})$. The resulting complementary DNA was diluted 40 times and RT-qPCR was performed using an ABI StepOne plus Real-Time PCR System, with 96-well optical reaction plates (both from Applied Biosystems; Thermo Fisher Scientific, Inc.). Amplification was performed at $95^{\circ} \mathrm{C}$ for $10 \mathrm{sec}$, followed by 40 cycles at $95^{\circ} \mathrm{C}$ for $5 \mathrm{sec}$, and $60^{\circ} \mathrm{C}$ for $20 \mathrm{sec}$. Gene expression levels were calculated with the $2^{-\Delta \Delta \mathrm{Cq}}$ method (38). The following primer sequences were used: GAPDH, forward 5'-GCTGAGTATGTCGTGGAGT-3' and reverse 5'-GTTCACACCCATCACAAAC-3'; Runx-2, forward 5'-CCC AGCCACCTTTACCTACA-3' and reverse 5'-TGGGAACTG ATAGGATGCTG-3'; ALP, forward 5'-CCGCCTGATCAAGTT CTCCT-3' and reverse 5'-TTCAGATGATCCATGCGGGG-3'; COL-1A1, forward 5'-CGTCAGCTCGTGTCCTGTGA-3' and reverse 5'-AGCTTGAGTAGCCATTGTCCA-3'; OPG, forward 5'-GTCCCTTGCCCTGACCACTCTT-3' and reverse 5'-AAC GCCCTTCCTCACACTCACA-3'; Osterix, forward 5'-CTG CAACTGGCTTTTCTGC-3' and reverse 5'-CAGCTCCTTAGG GCCACTT-3'; PPAR $\gamma$, forward 5'-CATCGAGGACATCCAAGA CA-3' and reverse 5'-TCTGTGACGATCTGCCTGAG-3'.

Western blot analysis. Proteins were obtained with the radioimmunoprecipitation assay lysis buffer (RIPA; Sigma-Aldrich; Merck KGaA) and subsequent centrifugation at 13,000 x $\mathrm{g}$ for $20 \mathrm{~min}$ at $4^{\circ} \mathrm{C}$. Protein concentration was determined by the BCA method. Proteins ( $20 \mu \mathrm{g}$ protein per lane) were separated by sodium dodecyl sulfate polyacrylamide gel electrophoresis (SDS-PAGE) on a $10 \%$ gel and transferred onto a nitrocellulose membrane (EMD Millipore). The membrane was blocked with 5\% skim milk in TBST (20 mM Tris- $\mathrm{HCl}, 150 \mathrm{mM}$ $\mathrm{NaCl}$, and $0.1 \%$ Tween 20, $\mathrm{pH} 7.5$ ) for at least $1 \mathrm{~h}$ and then incubated with each primary antibody overnight at $4^{\circ} \mathrm{C}$. The membranes were washed with TBST buffer at least five times for $5 \mathrm{~min}$ each, and incubated with the individual horseradish peroxidase (HRP)-conjugated secondary antibody $(1: 1,000$, cat. no. ab6721; Abcam) for $1 \mathrm{~h}$ at room temperature. The protein bands were visualized using an enhanced chemiluminescence (ECL) system (Amersham; GE Healthcare). Equal loading of samples was verified by immunoblotting of $\beta$-actin (Abcam) or GAPDH (Abcam) for total fraction, and TFIIB for nuclear fraction as previously described (39). The primary antibodies against OPG (1:1,000, cat. no. ab124820), COL-1 (1:500, cat. no. ab109025), ALP (1:1,000, ab83259), Osterix (1:500, cat. no. ab22552), Runx-2 (1:1,000, ab23981), PPAR $\gamma(1: 1,000$, cat. no. ab59256), p-GSK3 $\beta$ (1:500, cat. no. ab68476), GSK-3 $\beta$ (1:1,000, cat. no. ab32391), p- $\beta$-catenin (1:500, cat. no. ab27798), $\beta$-catenin $(1: 1,000$, ab223075), p-p38 MAPK $(1: 1,000$, cat. no. ab4822), p38 MAPK (1:1,000, cat. no. ab170099), and Wnt3a (1:500, cat. no. ab219412) were purchased from Abcam.
The expressions of targeted proteins were quantified by densitometry using ImageJ software (version 1.49; National Institutes of Health).

ADSC differentiation. ADSCs were induced to differentiate to osteogenic and adipogenic lineages as previously described (40). For osteogenic differentiation, confluent cells were incubated in Dulbecco's modified Eagle's medium (DMEM) with 10\% FBS (both from Gibco; Thermo Fisher Scientific, Inc.), $0.1 \mu \mathrm{M}$ dexamethasone (MCE, USA), $100 \mu \mathrm{g} / \mathrm{ml}$ ascorbate (MCE), and $10 \mathrm{mM} \beta$-glycerophosphate (Sigma-Aldrich; Merck KGaA). After 3 weeks, the cells were fixed in ice-cold $70 \%$ ethanol for $1 \mathrm{~h}$, washed with deionized water, and stained with $1 \%$ alizarin red (Sigma; Merck KGaA) for $5 \mathrm{~min}$ at $37^{\circ} \mathrm{C}$.

For adipogenic differentiation, ADSCs were cultured to confluence for 3 days and then incubated in DMEM with 10\% FBS, $10 \mathrm{ng} / \mathrm{ml}$ insulin (Sigma; Merck KGaA), $500 \mathrm{mM}$ 3-isobutyl-1-methylxanthine (Sigma; Merck KGaA), 1 mM dexamethasone (MCE), and $1 \mathrm{mM}$ rosiglitazone (Sigma; Merck $\mathrm{KGaA})$. After 3 weeks, the cells were fixed with $4 \%$ paraformaldehyde (PFA), washed with $60 \%$ isopropanol for $5 \mathrm{~min}$, and stained with oil red O (0.25\% wt/vol; Sigma; Merck KGaA) for $10 \mathrm{~min}$. After staining, cells were washed several times with deionized water.

Statistical analysis. Data are presented as the means \pm SD from at least three separate experiments. To assess the significance of differences between two groups, Student's t-test was performed. Results for more than two groups were evaluated by one-way ANOVA followed by Bonferroni's post hoc test. Statistical calculations were performed using SPSS 20.0 (IBM Corp., USA). A P-value of $<0.05$ indicated a statistically significant difference $\left({ }^{*} \mathrm{P}<0.05,{ }^{* *} \mathrm{P}<0.01\right.$, and ${ }^{* * *} \mathrm{P}<0.001$, as indicated in the figures).

\section{Results}

Exendin-4 facilitates ADSC-mediated bone repair. In order to elucidate the effect of exendin- 4 on bone defect repair, we previously established a bone-defect model in the distal metaphysis of the femur (34). Compared to the control group, the bone-defect group exhibited significantly decreased bone/tissue volume, trabecular bone thickness, and trabecular bone number, as well as a significant increase in trabecular bone space, indicating that the bone defect model was successfully established (Fig. 1A and B). When ADSCs were introduced into the wound, a significant increase in bone volume/tissue volume and trabecular bone score was observed compared to the non-treated bone-defect group (Fig. 1A and B), suggesting a role of ADSCs in repairing the defective bones. Notably, defective bones that received both ADSCs and exendin-4 markedly recovered, as evidenced by the bone volume/tissue volume, trabecular bone thickness, and trabecular bone space that were statistically similar to those of the non-injured control group (Fig. 1A and B). In addition, the mineral apposition status was visualized by calcein and tetracycline labeling, and the results revealed that defective bones that received both ADSCs and exendin-4 had an identical mineral apposition status to that of the control group (Fig. 1C). 
A

Con

bd

bd+ADSCs+EX-4

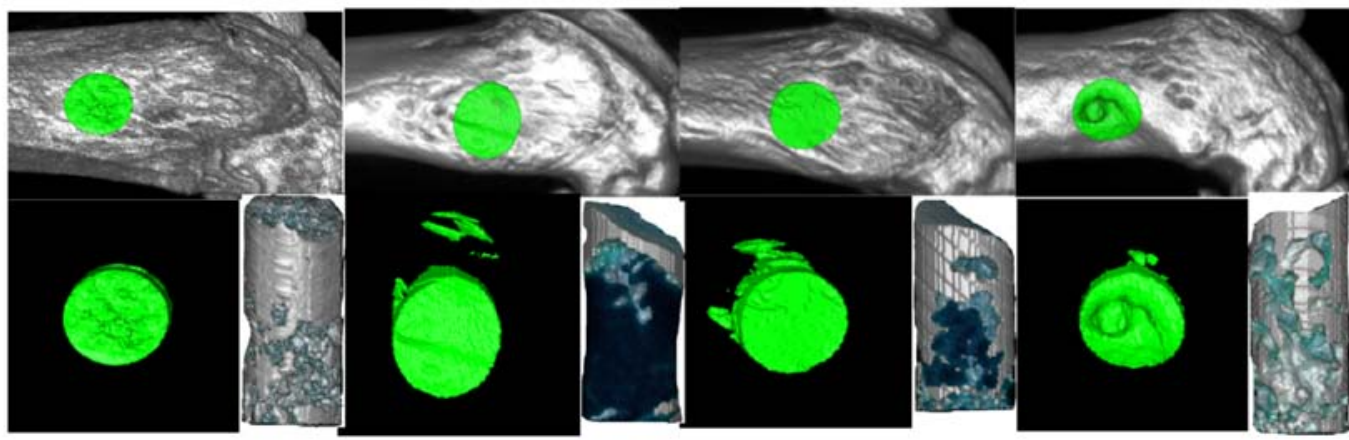

B

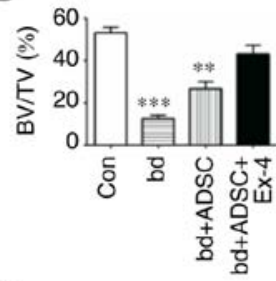

C

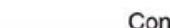

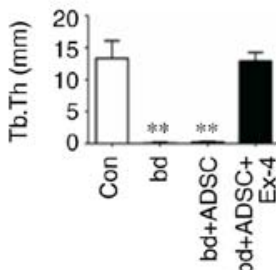

bd

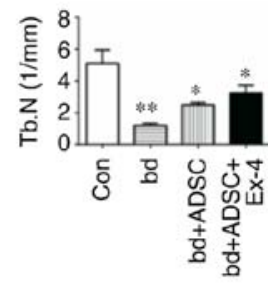

bd+ADSCs

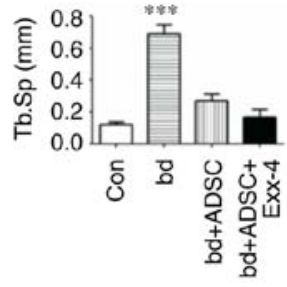

$b d+A D S C s+E X-4$

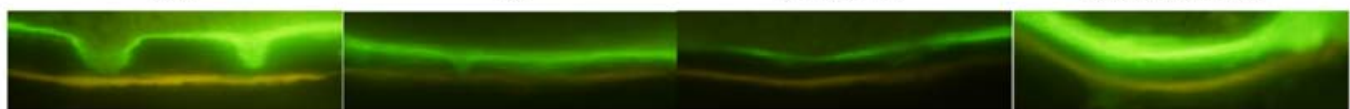

Figure 1. Exendin-4 facilitates bone defect repair. (A) Representative micro-computed tomography $(\mu \mathrm{CT})$ scanning results of control mice bone (Con; $\mathrm{n}=6)$, defective bone (bd; $n=6$ ), defective bone receiving ADSCs (bd+ADSCs; $n=6$ ), and defective bone receiving ADSCs and exendin-4 (bd+ADSCs+Ex-4; $n=6$ ). (B) Quantitative presentation of microarchitectural parameters of control mice bone (Con; $n=6$ ), defective bone (bd; $n=6)$, defective bone receiving ADSCs (bd+ADSCs; $n=6$ ), and defective bone receiving ADSCs and exendin-4 (bd+ADSCs+Ex-4; $n=6$ ). Error bars represent the mean $\pm \mathrm{SD}$. ${ }^{*} \mathrm{P}<0.05,{ }^{* *} \mathrm{P}<0.01$ and ${ }^{* * *} \mathrm{P}<0.001$. (C) Calcein and tetracycline double staining of control mice bone (Con; $\mathrm{n}=6$ ), defective bone (bd; $\mathrm{n}=6$ ), defective bone receiving ADSCs (bd+ADSCs; $n=6$ ), and defective bone receiving ADSCs and exendin-4 (bd+ADSCs+Ex-4; n=6). ADSCs, adipose-derived stem cells .
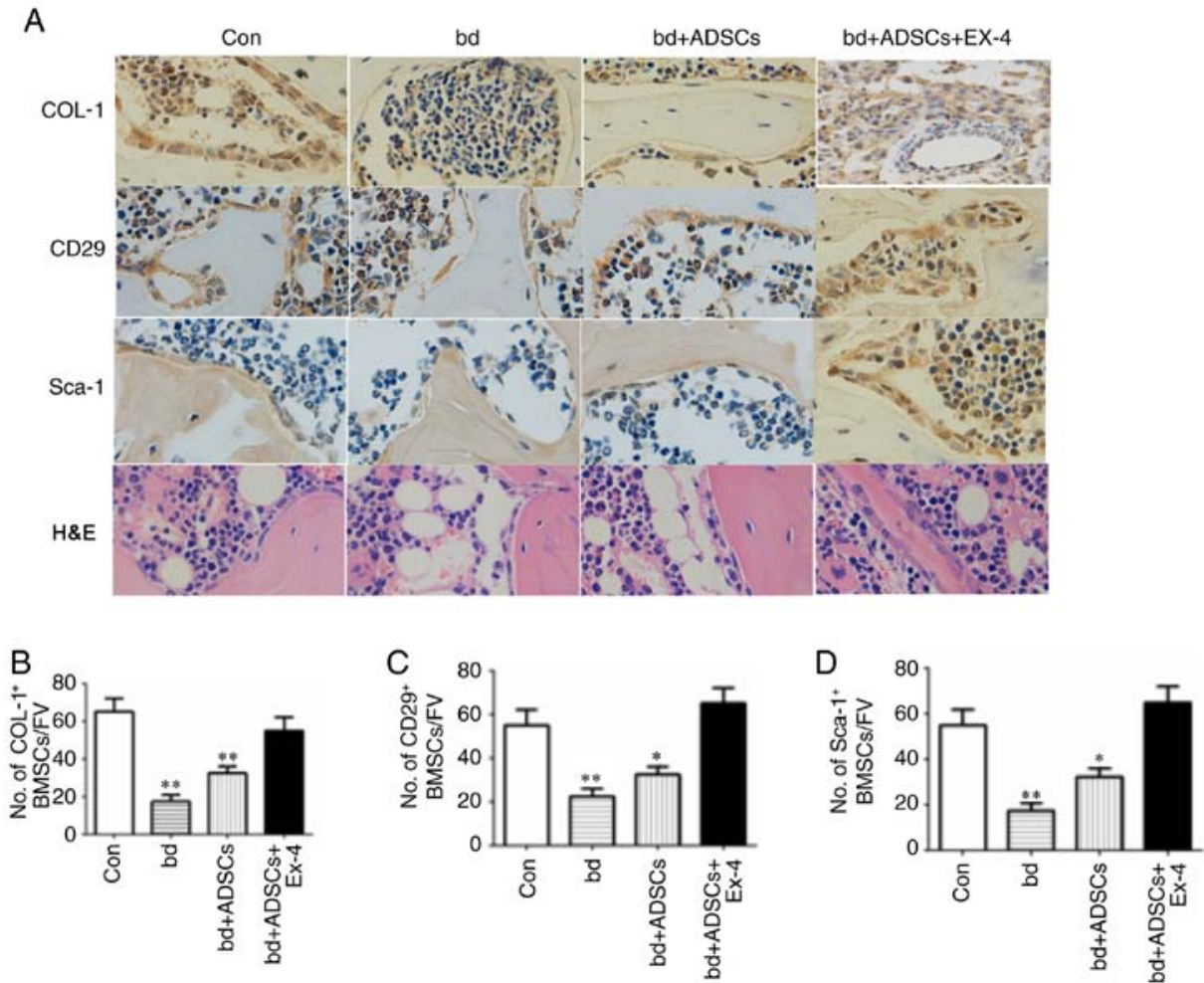

Figure 2. Exendin-4 promotes osteogenic differentiation and inhibits adipogenic differentiation. (A) Representative immunohistochemical results of COL-1, CD29, Sca-1, and H\&E staining in bones with different treatments. Magnification, x200. (B) Quantification of COL-1-positive cells. No. of OB/FV, number of osteoblasts/field of view. (C) Quantification of CD29-positive cells. No. of OB/FV, number of osteoblasts/field of view. Error bar represent the mean \pm SD (D) Quantification of Sca-1-positive cells. No. of OB/FV, number of osteoblasts/field of view. Error bar represent mean \pm SD. ${ }^{*} \mathrm{P}<0.05$ and ${ }^{* *} \mathrm{P}<0.01 \mathrm{vs}$. the Con group. COL-1, collagen 1; ADSCs, adipose-derived stem cells. 

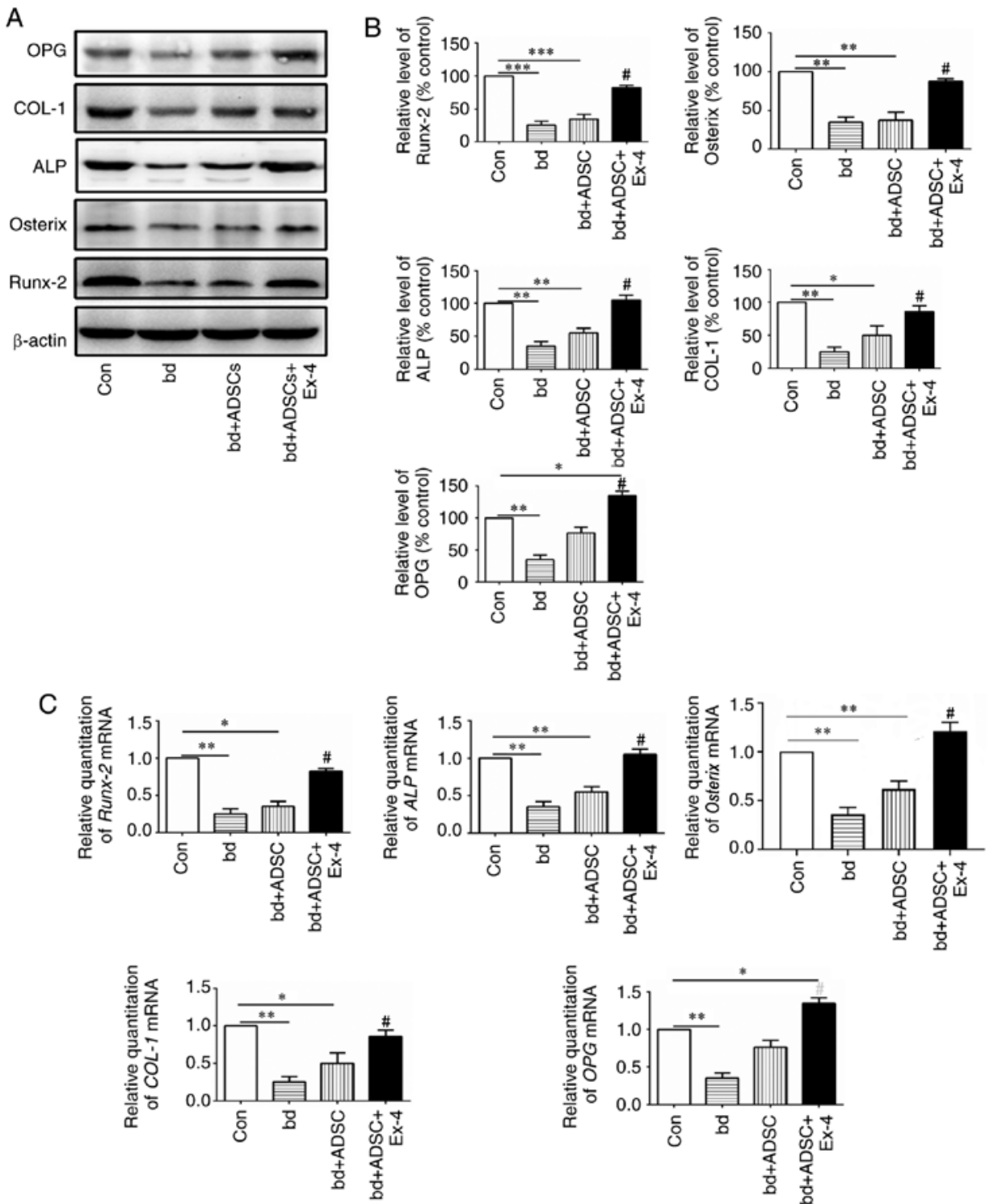

Figure 3. Exendin-4 induces expression of genes related to bone formation. (A) Protein expression levels of bone formation-related genes in bones with different treatments. (B) Quantification results of the protein expression levels of bone formation-related genes in bones administered different treatments. (C) mRNA expression levels of bone formation-related genes in bones administered different treatments. ${ }^{*} \mathrm{P}<0.05$, ${ }^{* *} \mathrm{P}<0.01$ and ${ }^{* * *} \mathrm{P}<0.001$ vs. control; ${ }^{\#} \mathrm{P}<0.05$ vs. bd group. OPG, osteoprotegerin; COL-1, collagen 1; ALP, alkaline phosphatase; ADSCs, adipose-derived stem cells.

Exendin-4 promotes the generation of osteoblasts during bone defect repair. To reveal the cellular mechanism through which exendin-4 facilitates bone repair, the osteogenic and adipogenic differentiation in defective bones subjected to ADSC and exendin-4 treatments were analyzed using immunohistochemical staining. Positive staining for COL-1 was significantly decreased in the defective bones, while ADSC administration improved the level of COL-1-positive staining. Notably, COL-1-positive signals in the defective bones treated with ADSCs and exendin-4 were comparable to those of the control group (Fig. 2A and B). The number of ADSCs were also examined in the defective bones after various treatments by immunohistochemical staining for CD29 and Sca-1, two molecular markers of ADSCs. The present results revealed a reduced number of ADSCs in defective bones, while exendin-4 treatment significantly attenuated the loss of ADSCs resulting from bone defects (Fig. 2C and D). Concurrently, H\&E staining revealed that the number of adipocytes was reduced in the defective bones treated with ADSCs and exendin-4 (Fig. 2A). Collectively, the present results revealed that exendin- 4 promoted the generation of osteoblasts and inhibited the generation of adipocytes.

Exendin-4 induces the expression of genes related to osteogenic differentiation. Since exendin-4 treatment significantly promoted osteogenic differentiation, the expression of genes related to osteogenic differentiation at both the protein and mRNA levels were next examined. The protein levels of osteoprotegerin (OPG), COL-1, alkaline phosphatase (ALP), Osterix, and Runx-2, all of which are closely related to bone metabolism, were first assessed (41-45). The present results revealed that the protein levels of OPG, COL-1, ALP, Osterix, and Runx-2 were significantly decreased in the defective bones, and ADSC treatment had little effect on the expression of these proteins in the defective bones. However, when the defective bones received both ADSCs and exendin- 4 treatment, the 
A
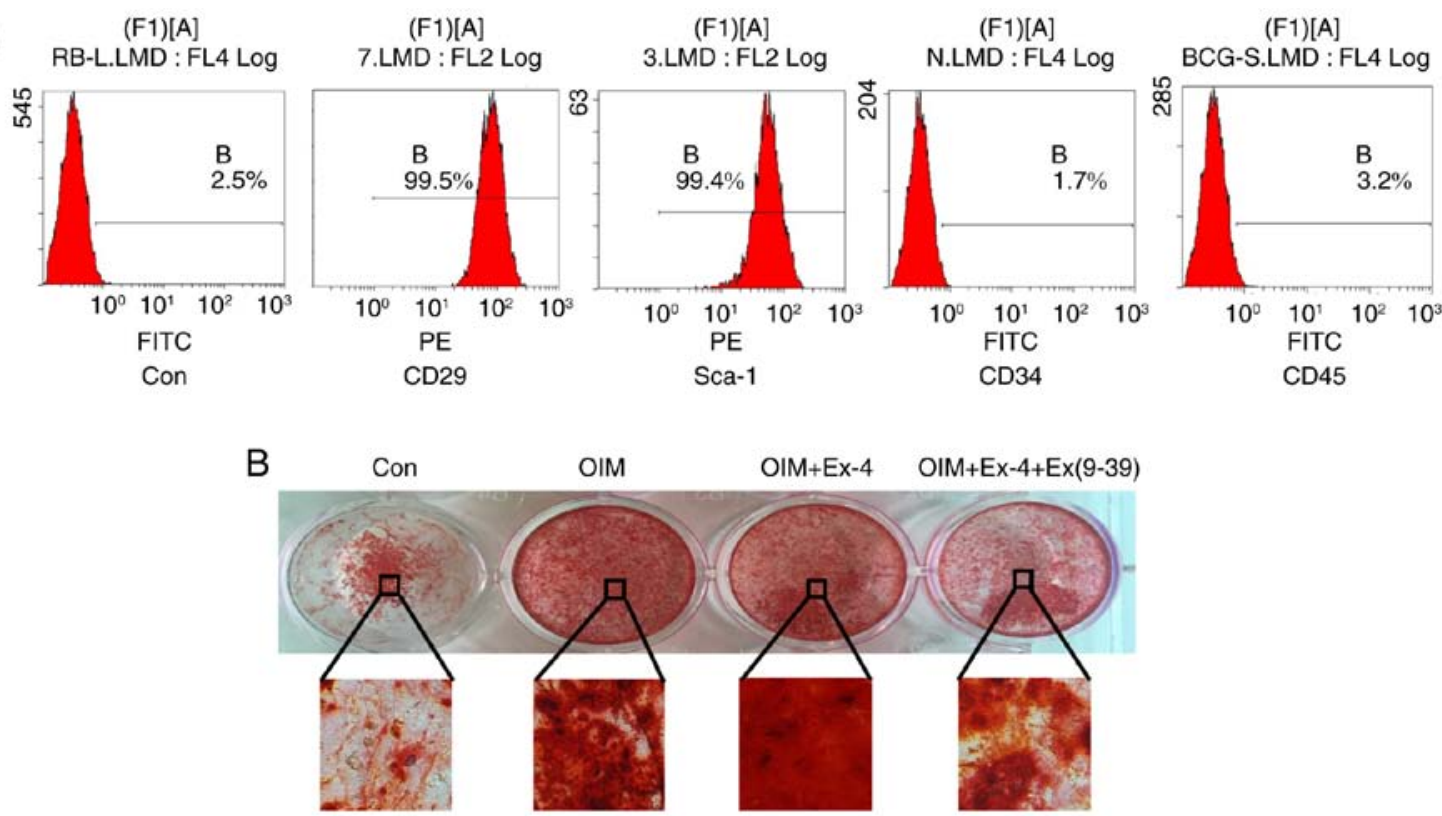

C

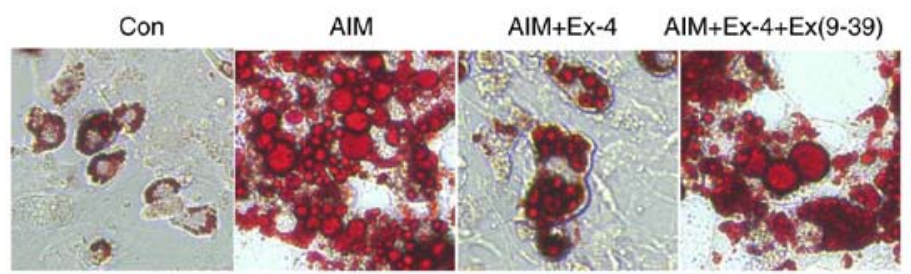

Figure 4. Exendin-4 promotes osteogenic differentiation in vitro. (A) Immuno-characterization of FACS-sorted. Sorted cells revealed high expression levels of ADSC-positive markers CD29 and Sca-1 and were negative for CD34 and CD45. (B) In vitro induction of osteogenic differentiation with ADSCs. Magnification, x200. (C) In vitro induction of adipogenic differentiation with ADSCs. Magnification, x200. ADSCs, adipose-derived stem cells; OIM, osteoblast-inducing medium; Ex (9-39), an antagonist of exendin-4; AIM, adipose-induced medium.

decrease in protein levels of OPG, COL-1, ALP, Osterix, and Runx-2 was significantly abolished (Fig. $3 \mathrm{~A}$ and $\mathrm{B}, \mathrm{P}<0.05$ vs. the bd group). Notably, it was observed that ADSC supplementation alone rescued the expression of $\mathrm{OPG}$, and administration of exendin-4 further increased the protein levels of OPG (Fig. 3A and B). These data indicated that administration of exendin-4 increased the protein levels of genes related to osteogenic differentiation. The mRNA levels of OPG, COL-1, ALP, Osterix, OPG, and Runx-2 were also examined, and it was revealed that they were in agreement with the protein levels of the respective genes (Fig. 3C).

Exendin-4 promotes osteogenic differentiation in vitro. To further validate the pro-osteogenic role of exendin-4, ADSCs were isolated and their differentiation in vitro was examined under exendin-4 treatment. First, the characteristics of cultured ADSCs were verified using flow cytometry, and the results revealed that the cells were positive for the mesenchymal stem cell marker CD29 and the ADSC marker Sca-1. Concurrently, the in vitro-cultured cells were negative for CD34 and CD45, indicating that the isolated cells maintained ADSC characteristics (Fig. 4A). Then in vitro osteoblast differentiation was induced with osteoblast induction medium (OIM). The results revealed that administration of exendin- 4 increased the osteogenic differentiation in vitro and notably, when exendin-4 was antagonized by its specific antagonist Ex (9-39), the osteogenic differentiation of ADSCs in vitro was significantly impaired (Fig. 4B). In addition, the adipogenic differentiation was also induced with adipose induction medium (AIM) in vitro. The results revealed that adipogenic differentiation was markedly inhibited by exendin-4. However, when exendin- 4 was antagonized by Ex (9-39), adipogenic differentiation increased (Fig. 4C).

The expression levels of genes related to osteogenic differentiation were next examined in the in vitro-cultured ADSCs. As anticipated, the expression levels of the relevant genes, OPG, COL-1, ALP, Osterix, and Runx-2, were all increased at both the protein and the mRNA levels when the cells were treated with exendin-4 (Fig. 5A-C). Concurrently, peroxisome proliferator-activated receptor-g (PPAR $\gamma)$, a molecular marker that indicates adipogenic differentiation, was significantly downregulated at both the mRNA and protein level when ADSCs were treated with exendin-4 (Fig. 5A-C). Collectively, the present data indicated that exendin- 4 promoted osteogenic differentiation and inhibited adipogenic differentiation in vitro.

To further understand how exendin-4 regulates ADSCs, the levels of GSK3 $\beta$, phosphorylated (p)-GSK3 $\beta$, p38 MAPK, p-p38 MAPK, $\beta$-catenin, $p$ - $\beta$-catenin, and Wnt3a were examined. Notably, administration of exendin-4 significantly increased the levels of Wnt3a and $\beta$-catenin, as well as nuclear localization of $\beta$-catenin (Fig. 6A-C), which corresponds to previous study (46). Moreover, the present results revealed that 
A

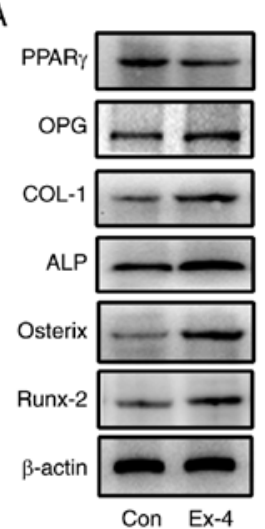

C
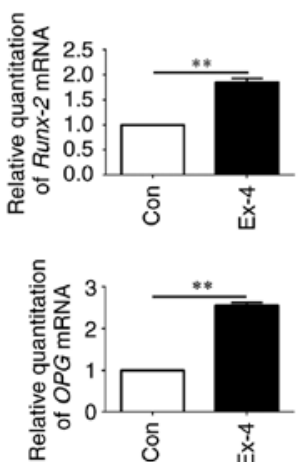

B
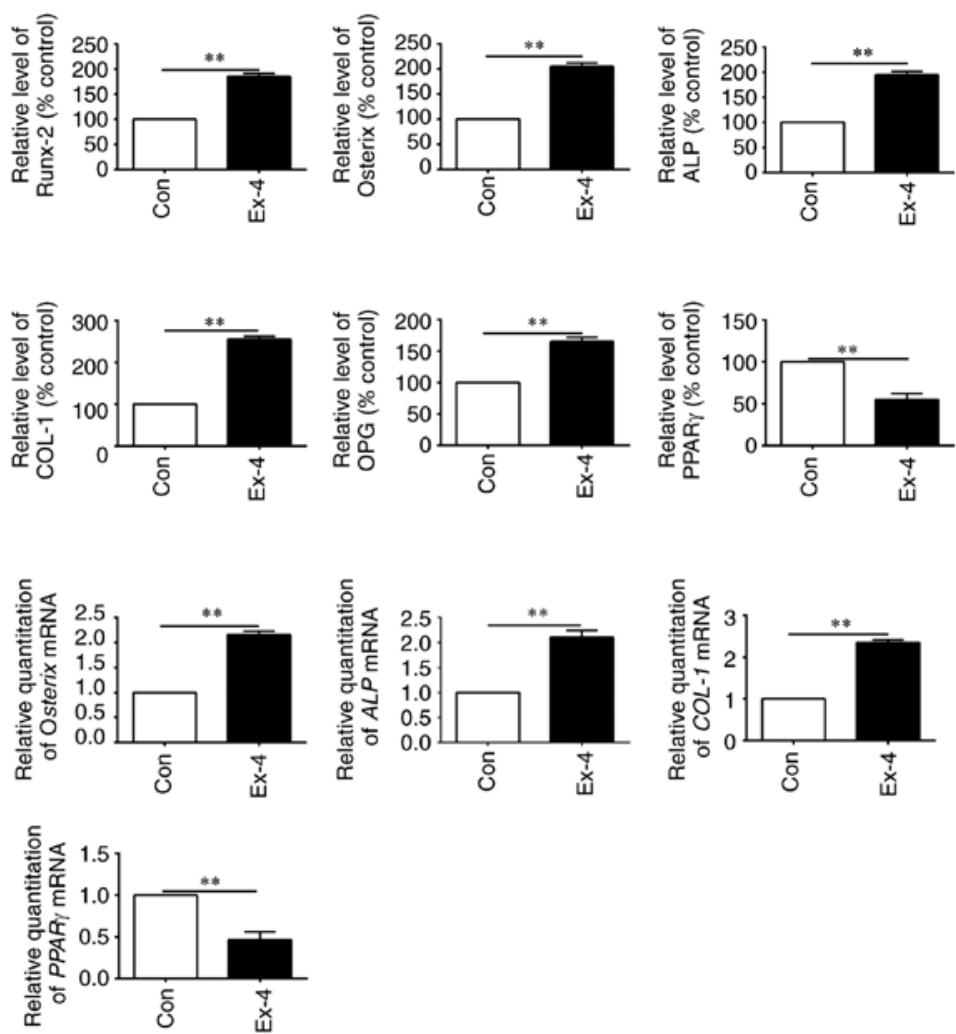

Figure 5. In vitro induction of bone formation-related genes by exendin-4. (A) Protein expression levels of bone formation-related genes from in vitro-cultured ADSCs administered different treatments. (B) Quantification results of the protein expression levels of bone formation-related genes from in vitro-cultured ADSCs administered different treatments. (C) mRNA expression levels of bone formation-related genes (OPG, COL-1, ALP, Osterix, PPAR $\gamma$ and Runx-2) from in vitro-cultured ADSCs administered different treatments. ADSCs, adipose-derived stem cells; OPG, osteoprotegerin; COL-1, collagen 1; ALP, alkaline phosphatase; PPAR $\gamma$, peroxisome proliferator-activated receptor- $\gamma$. ${ }^{* *} \mathrm{P}<0.01$.

A

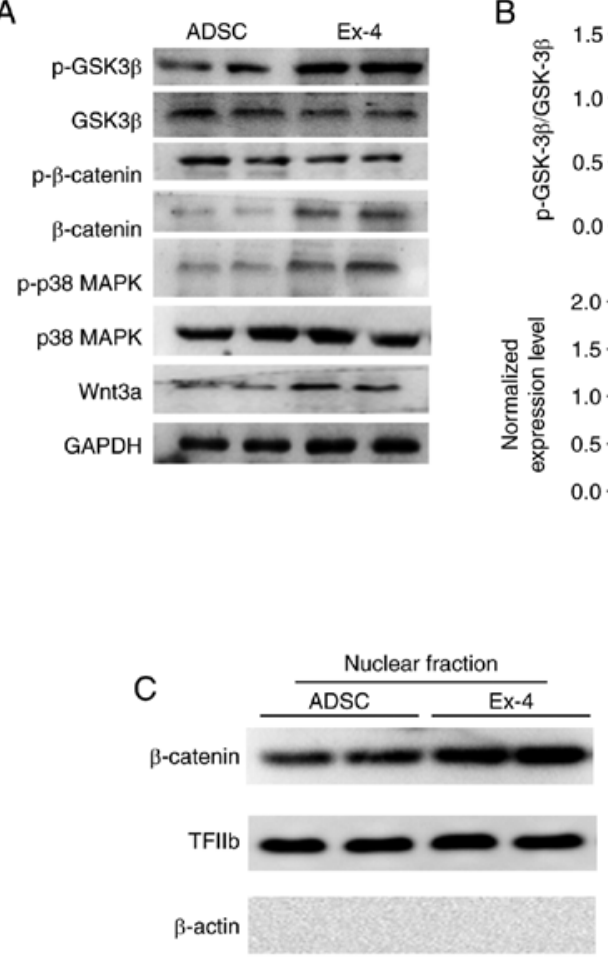

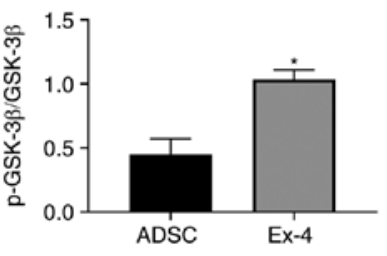
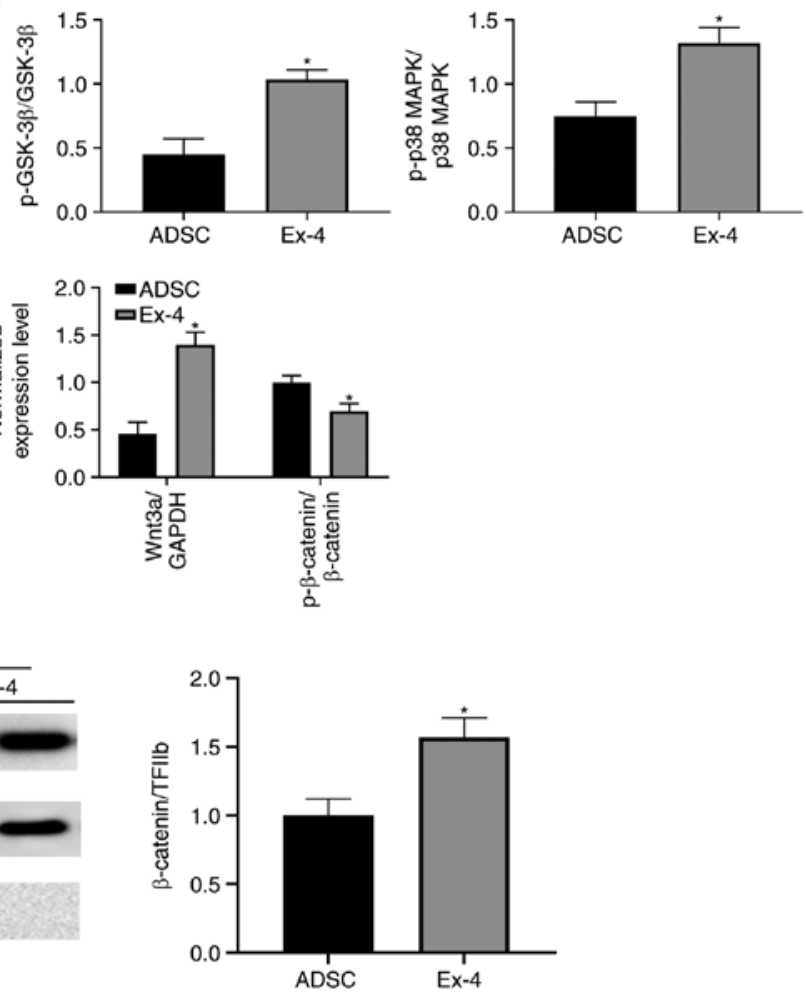

Figure 6. Exendin-4 promotes ADSC differentiation through PI3K and Wnt signaling pathways. (A) The levels of relative proteins in the ADSCs were determined by western blotting. (B) Quantitative results were normalized against GAPDH levels. Values are presented as the group mean \pm SEM ( $\mathrm{n}=3$ ). (C) Nuclear localization of $\beta$-catenin. " $\mathrm{P}<0.05$ vs. the ADSC group. ADSC, adipose-derived stem cell. 
p-p38 MAPK and p-GSK3 $\beta$ were significantly upregulated when ADSCs were treated with exendin-4 (Fig. 6A and B) indicating that exendin-4 exhibited identical function as in the neuronal system $(47,48)$. These alterations in critical signaling pathways may induce indirect regulation of the expression of genes related to osteogenic differentiation. Hence, the present data demonstrated that multiple signaling pathways were involved in exendin-4-mediated ADSC differentiation.

\section{Discussion}

A key to the successful application of ADSCs in bone tissue regeneration is to accurately adjust the cell differentiation after effective delivery. In the present study, the glucagon-like peptide 1 receptor agonist exendin-4, which promoted osteogenic differentiation of ADSCs both in vitro and in vivo was characterized and the transcription of genes related to osteogenic differentiation was induced. The present results revealed that exendin- 4 facilitated bone repair in mice injected with ADSCs after a large-scale bone defect in vivo, indicating that exendin- 4 is a promising candidate for assisting in the repair of bone defects using adult stem cells.

Several types of adult stem cells have demonstrated potential in bone tissue engineering $(5,7)$, however, ADSCs display multiple advantages among the various types of adult stem cells. The abundance of ADSCs is higher than that of other adult stem cells and it is easier to obtain these cells $(49,50)$. The role of ADSCs in promoting bone regeneration has been revealed for a long time $(51,52)$, and several supplements that were used to induce osteogenic differentiation of BMSCs also promoted osteogenic differentiation of ADSCs in vitro (53-55). In the present study, it was demonstrated that exendin-4, a clinically approved drug for type 2 diabetes treatment, facilitated the osteogenic differentiation of ADSCs both in vivo and in vitro. In a rat bone loss model caused by mechanical unloading, exendin- 4 was revealed to improve bone mass and bone strength during the natural bone healing process (32). In exendin-4-induced bone reconstruction, a significant increase in osteoblasts generated by BMSC was observed (32). The present results further revealed the pro-osteogenic role of exendin-4 in ADSC-based bone repair, which not only agreed with previous research but further demonstrated a clinical application prospect for exendin-4 in tissue engineering-based bone repair.

Exendin-4 promotes osteogenic differentiation through various molecular mechanisms. The present results revealed that both mRNA and protein levels of Runx-2, Osterix, APL, COL-1, and OPG were significantly increased in the regenerating bones in situ (Fig. 3). However, the addition of ADSCs alone did not result in a marked increase in Runx-2 and Osterix at the protein level, suggesting that exendin-4 is partially required to trigger a complete osteogenic differentiation. Although, evidence to illustrate the detailed mechanism of how these genes were transcriptionally activated was not obtained, the present results revealed that multiple critical signaling pathways were altered when exendin- 4 was administered, including GSK3 $\beta$, MAPK, and Wnt $/ \beta$-catenin signaling. These signals have been revealed to promote osteogenic differentiation by increasing the expression of genes including Runx-2, Osterix, APL, COL-1, and OPG (56). These alterations may indirectly contribute to the expression changes of genes related to osteogenic differentiation. Notably, the present data also indicated that the induction of osteogenic differentiation by exendin- 4 depends on transcriptional activation of genes related to osteogenic differentiation (Fig. 3). Thus, it is crucial to characterize the downstream signaling activated by exendin-4 in ADSCs to further understand the detailed molecular mechanism and guide clinical applications. Meng et al suggested that exendin- 4 treatment induced the expression and nuclear localization of $\beta$-catenin in BMSCs, which in turn further directed the differentiation of BMSCs (32). In addition, the present data also revealed that exendin- 4 significantly promoted osteogenic differentiation and inhibited adipogenic differentiation in vitro, providing a unified cellular model to study the underlying molecular mechanisms.

Since exendin- 4 has been approved as an orally administered medication, its use in conjunction with genetically modified stem cells and other bio-active molecules provides new perspectives for bone healing. Jin et al reported that the ERK signaling pathway balanced the osteogenesis and adipogenesis of ADSCs during bone defect repair (14). Moreover, epigenetic modifications and basic fibroblast growth factor (bFGF) signaling were also revealed to be critical to improve bone defect repair using BMSCs $(57,58)$. The combined use of inhibitors or agonists of these signaling pathways may provide fine tuning of ADSC proliferation and differentiation, which may finally satisfy the needs of various bone repair technologies. Recent studies also suggested that miR-146a-regulated osteogenesis of ADSCs can be adjusted through the BMP signaling pathway during bone regeneration (59). Furthermore, miR-342-3p was reported to increase osteogenic differentiation of umbilical cord mesenchymal stem cells (60). Based on these findings, it will be of great significance to combine exendin- 4 and miRNAs to develop practical therapies for bone regeneration.

In summary, the present results demonstrated that exendin- 4 promoted the osteogenic differentiation of transplanted ADSCs in situ, which in turn repaired the defective bones. In addition, exendin- 4 inhibited the adipogenic differentiation of transplanted ADSCs to guarantee eventual bone formation. The present data further revealed the clinical potential of ADSCs in tissue engineering-based bone defect repair and characterized the role of exendin- 4 in facilitating the osteogenic differentiation of transplanted ADSCs.

\section{Acknowledgements}

Not applicable.

\section{Funding}

The present study was financially supported by the National Natural Science Foundation of China (grant nos. 81771107 and 81470775).

\section{Availability of data and materials}

The datasets used and/or analyzed for the current study are available from the corresponding author upon reasonable request. 


\section{Authors' contributions}

BLD and WZZ performed the statistical analyses, evaluated the results, and drafted the study. YLS, BLD and WZZ participated in the conception and design of the study. YSD, YQH and XFC contributed to data collection and interpretation, and manuscript revision. SS and ZY contributed to analyzing data. All authors have read and approved the final manuscript.

\section{Ethics approval and consent to participate}

All animal experiments were performed in accordance with the guidelines of the NIH (Publication no. 85e23 Rev. 1985) and were approved by the Animal Care and Use Committee of The Fourth Military Medical University.

\section{Patient consent for publication}

Not applicable.

\section{Competing interests}

The authors declare that they have no competing interests.

\section{References}

1. Dufrane D: Impact of age on human adipose stem cells for bone tissue engineering. Cell Transplant 26: 1496-1504, 2017.

2. Weinand C, Neville CM, Weinberg E, Tabata Y and Vacanti JP: Optimizing biomaterials for tissue engineering human bone using mesenchymal stem cells. Plast Reconstr Surg 137: 854-863, 2016.

3. Bertolo A, Mehr M, Janner-Jametti T, Graumann U, Aebli N, Baur M, Ferguson SJ and Stoyanov JV: An in vitro expansion score for tissue-engineering applications with human bone marrow-derived mesenchymal stem cells. J Tissue Eng Regen Med 10: 149-161, 2016.

4. Alizadeh A, Moztarzadeh F, Ostad SN, Azami M, Geramizadeh B Hatam G, Bizari D, Tavangar SM, Vasei M and Ai J: Synthesis of calcium phosphate-zirconia scaffold and human endometrial adult stem cells for bone tissue engineering. Artif Cells Nanomed Biotechnol 44: 66-73, 2016.

5. Wang P, Liu X, Zhao L, Weir MD, Sun J, Chen W, Man Y and $\mathrm{Xu} \mathrm{HH}$ : Bone tissue engineering via human induced pluripotent, umbilical cord and bone marrow mesenchymal stem cells in rat cranium. Acta Biomater 18: 236-248, 2015.

6. Zuk PA, Zhu M, Mizuno H, Huang J, Futrell JW, Katz AJ Benhaim P, Lorenz HP and Hedrick MH: Multilineage cells from human adipose tissue: Implications for cell-based therapies. Tissue Eng 7: 211-228, 2001.

7. Amini AR, Laurencin CT and Nukavarapu SP: Bone tissue engineering: Recent advances and challenges. Crit Rev Biomed Eng 40: 363-408, 2012.

8. Oryan A, Alidadi S, Moshiri A and Maffulli N: Bone regenerative medicine: Classic options, novel strategies, and future directions. J Orthop Surg Res 9: 18, 2014.

9. Oryan A, Kamali A, Moshiri A and Baghaban Eslaminejad M: Role of mesenchymal stem cells in bone regenerative medicine: What is the evidence? Cells Tissues Organs 204: 59-83, 2017.

10. Tapp H, Hanley EN Jr, Patt JC and Gruber HE: Adipose-derived stem cells: Characterization and current application in orthopaedic tissue repair. Exp Biol Med (Maywood) 234: 1-9, 2009.

11. Caplan AI and Dennis JE: Mesenchymal stem cells as trophic mediators. J Cell Biochem 98: 1076-1084, 2006.

12. Orbay H, Busse B, Leach JK and Sahar DE: The effects of Adipose-derived stem cells differentiated into endothelial cells and osteoblasts on healing of critical size calvarial defects. J Craniofac Surg 28: 1874-1879, 2017.

13. Knippenberg M, Helder MN, Zandieh Doulabi B, Wuisman PI and Klein-Nulend $\mathrm{J}$ : Osteogenesis versus chondrogenesis by BMP-2 and BMP-7 in adipose stem cells. Biochem Biophys Res Commun 342: 902-908, 2006.
14. Jin Y, Zhang W, Liu Y, Zhang M, Xu L, Wu Q, Zhang X, Zhu Z, Huang $Q$ and Jiang X: rhPDGF-BB via ERK pathway osteogenesis and adipogenesis balancing in ADSCs for critical-sized calvarial defect repair. Tissue Eng Part A 20: 3303-3313, 2014.

15. Lough DM, Chambers C, Germann G, Bueno R, Reichensperger J, Swanson E, Dyer M, Cox L, Harrison C and Neumeister MW: Regulation of ADSC Osteoinductive potential using notch pathway inhibition and gene rescue: A Potential On/Off Switch for clinical applications in bone formation and reconstructive efforts. Plast Reconstr Surg 138: 642e-652e, 2016.

16. Li S, Hu C, Li J, Liu L, Jing W, Tang W, Tian W and Long J: Effect of miR-26a-5p on the $\mathrm{Wnt} / \mathrm{Ca}(2+)$ pathway and osteogenic differentiation of mouse Adipose-derived mesenchymal stem cells. Calcif Tissue Int 99: 174-186, 2016.

17. Hu X, Fu Y, Zhang X, Dai L, Zhu J, Bi Z, Ao Y and Zhou C: Histone deacetylase inhibitor sodium butyrate promotes the osteogenic differentiation of rat adipose-derived stem cells. Dev Growth Differ 56: 206-213, 2014.

18. Gersbach CA, Byers BA, Pavlath GK and Garcia AJ: Runx2/Cbfa1 stimulates transdifferentiation of primary skeletal myoblasts into a mineralizing osteoblastic phenotype. Exp Cell Res 300: 406-417, 2004.

19. Lee B, Thirunavukkarasu K, Zhou L, Pastore L, Baldini A, Hecht J, Geoffroy V, Ducy P and Karsenty G: Missense mutations abolishing DNA binding of the osteoblast-specific transcription factor OSF2/CBFA1 in cleidocranial dysplasia. Nat Genet 16 : 307-310, 1997.

20. Zhang X, Yang M, Lin L, Chen P, Ma KT, Zhou CY and Ao YF: Runx2 overexpression enhances osteoblastic differentiation and mineralization in adipose-derived stem cells in vitro and in vivo. Calcif Tissue Int 79: 169-178, 2006.

21. Basoli V, Santaniello S, Cruciani S, Ginesu GC, Cossu ML, Delitala AP, Serra PA, Ventura C and Maioli M: Melatonin and Vitamin D interfere with the adipogenic fate of Adipose-derived stem cells. Int J Mol Sci 18: E981, 2017.

22. Malhotra R, Singh L, Eng J and Raufman JP: Exendin-4, a new peptide from Heloderma suspectum venom, potentiates cholecystokinin-induced amylase release from rat pancreatic acini. Regul Pept 41: 149-156, 1992.

23. Eng J,Kleinman WA, Singh L, Singh G and Raufman JP: Isolation and characterization of exendin-4, an exendin-3 analogue, from Heloderma suspectum venom. Further evidence for an exendin receptor on dispersed acini from guinea pig pancreas. J Biol Chem 267: 7402-7405, 1992.

24. Eng J: Exendin peptides. Mt Sinai J Med 59: 147-149, 1992.

25. Edwards CM, Stanley SA, Davis R, Brynes AE, Frost GS, Seal LJ, Ghatei MA and Bloom SR: Exendin-4 reduces fasting and postprandial glucose and decreases energy intake in healthy volunteers. Am J Physiol Endocrinol Metab 281: E155-E161, 2001.

26. Nielsen LL, Young AA and Parkes DG: Pharmacology of exenatide (synthetic exendin-4): A potential therapeutic for improved glycemic control of type 2 diabetes. Regul Pept 117: 77-88, 2004.

27. Yamada C, Yamada Y, Tsukiyama K, Yamada K, Udagawa N, Takahashi N, Tanaka K, Drucker DJ, Seino Y and Inagaki N: The murine glucagon-like peptide-1 receptor is essential for control of bone resorption. Endocrinology 149: 574-579, 2008.

28. Nuche-Berenguer B, Lozano D, Gutierrez-Rojas I, Moreno P, Mariñoso ML, Esbrit P and Villanueva-Peñacarrillo ML: GLP-1 and exendin-4 can reverse hyperlipidic-related osteopenia. J Endocrinol 209: 203-210, 2011.

29. Ma X, Meng J, Jia M, Bi L, Zhou Y, Wang Y, Hu J, He G and Luo X: Exendin-4, a glucagon-like peptide-1 receptor agonist, prevents osteopenia by promoting bone formation and suppressing bone resorption in aged ovariectomized rats. J Bone Miner Res 28: 1641-1652, 2013.

30. Feng Y, Su L, Zhong X, Guohong W, Xiao H, Li Y and Xiu L: Exendin-4 promotes proliferation and differentiation of MC3T3-E1 osteoblasts by MAPKs activation. J Mol Endocrinol 56: 189-199, 2016.

31. He J, Wang C, Sun Y, Lu B, Cui J, Dong N, Zhang M, Liu Y and Yu B: Exendin-4 protects bone marrow-derived mesenchymal stem cells against oxygen/glucose and serum deprivation-induced apoptosis through the activation of the cAMP/PKA signaling pathway and the attenuation of ER stress. Int J Mol Med 37: 889-900, 2016.

32. Meng J, Ma X, Wang N, Jia M, Bi L, Wang Y, Li M, Zhang H, Xue X, Hou Z, et al: Activation of GLP-1 receptor promotes bone marrow stromal cell osteogenic differentiation through $\beta$-Catenin. Stem Cell Reports 6: 579-591, 2016. 
33. Shen WR, Kimura K, Ishida M, Sugisawa H, Kishikawa A, Shima K, Ogawa S, Qi J and Kitaura H: The glucagon-like Peptide-1 receptor agonist Exendin-4 inhibits lipopolysaccharide-induced osteoclast formation and bone resorption via inhibition of TNF- $\alpha$ expression in macrophages. J Immunol Res 2018: 5783639, 2018.

34. Uusitalo H, Rantakokko J, Ahonen M, Jämsä T, Tuukkanen J, KäHäri V, Vuorio E and Aro HT: A metaphyseal defect model of the femur for studies of murine bone healing. Bone 28: 423-429, 2001.

35. Lin M, Liu R, Gozal D, Wead WB, Chapleau MW, Wurster R and Cheng ZJ: Chronic intermittent hypoxia impairs baroreflex control of heart rate but enhances heart rate responses to vagal efferent stimulation in anesthetized mice. Am J Physiol Heart Circ Physiol 293: H997-H1006, 2007.

36. Soranno DE, Rodell CB, Altmann C, Duplantis J, Andres-Hernando A, Burdick JA and Faubel S: Delivery of interleukin-10 via injectable hydrogels improves renal outcomes and reduces systemic inflammation following ischemic acute kidney injury in mice. Am J Physiol Renal Physiol 311: F362-F372, 2016

37. Wang N, Gao J, Jia M, Ma X, Lei Z, Da F, Yan F, Zhang H, Zhou Y, Li M, et al: Exendin-4 induces bone marrow stromal cells migration through bone marrow-derived macrophages polarization via PKA-STAT3 signaling pathway. Cell Physiol Biochem 44: 1696-1714, 2017.

38. Livak KJ and Schmittgen TD: Analysis of relative gene expression data using real-time quantitative PCR and the 2(-Delta Delta C(T)) method. Methods 25: 402-408, 2001.

39. Zhang N, Wei P, Gong A, Chiu WT, Lee HT, Colman H, Huang H, Xue J, Liu M, Wang Y, et al: FoxM1 promotes $\beta$-catenin nuclear localization and controls Wnt target-gene expression and glioma tumorigenesis. Cancer Cell 20: 427-442, 2011.

40. Roxburgh J, Metcalfe AD and Martin YH: The effect of medium selection on adipose-derived stem cell expansion and differentiation: Implications for application in regenerative medicine. Cytotechnology 68: 957-967, 2016.

41. Simonet WS, Lacey DL, Dunstan CR, Kelley M, Chang MS, Lüthy R, Nguyen HQ, Wooden S, Bennett L, Boone T, et al: Osteoprotegerin: A novel secreted protein involved in the regulation of bone density. Cell 89: 309-319, 1997.

42. Gerstenfeld LC and Shapiro FD: Expression of bone-specific genes by hypertrophic chondrocytes: Implication of the complex functions of the hypertrophic chondrocyte during endochondral bone development. J Cell Biochem 62: 1-9, 1996.

43. Greep RO, Fischer CJ and Morse A: Alkaline phosphatase in odontogenesis and osteogenesis and its histochemical demonstration after demineralization. J Am Dent Assoc 36: 427-442, 1948.

44. Nakashima K, Zhou X, Kunkel G, Zhang Z, Deng JM, Behringer RR and de Crombrugghe $B$ : The novel zinc finger-containing transcription factor osterix is required for osteoblast differentiation and bone formation. Cell 108: 17-29, 2002.

45. McCarthy TL, Ji C, Chen Y, Kim KK, Imagawa M, Ito Y and Centrella M: Runt domain factor (Runx)-dependent effects on CCAAT/ enhancer-binding protein delta expression and activity in osteoblasts. J Biol Chem 275: 21746-21753, 2000.

46. Seo MH, Lee J, Hong SW, Rhee EJ, Park SE, Park CY, Oh KW, Park SW and Lee WY: Exendin-4 inhibits hepatic lipogenesis by increasing beta-catenin signaling. PLoS One 11: e0166913, 2016

47. Xu W, Yang Y, Yuan G, Zhu W, Ma D and Hu S: Exendin-4, a glucagon-like peptide-1 receptor agonist, reduces Alzheimer disease-associated tau hyperphosphorylation in the hippocampus of rats with type 2 diabetes. J Investig Med 63: 267-272, 2015.
48. Hayes MR: Neuronal and intracellular signaling pathways mediating GLP-1 energy balance and glycemic effects. Physiol Behav 106: 413-416, 2012

49. Mainil-Varlet P, Aigner T, Brittberg M, Bullough P, Hollander A, Hunziker E, Kandel R, Nehrer S, Pritzker K, Roberts S, et al: Histological assessment of cartilage repair: A report by the Histology endpoint committee of the international cartilage repair society (ICRS). J Bone Joint Surg Am 85-A (Suppl 2): S45-S57, 2003.

50. Veronesi F, Maglio M, Tschon M, Aldini NN and Fini M: Adipose-derived mesenchymal stem cells for cartilage tissue engineering: State-of-the-art in in vivo studies. J Biomed Mater Res A 102: 2448-2466, 2014

51. Di Bella C, Farlie P and Penington AJ: Bone regeneration in a rabbit critical-sized skull defect using autologous adipose-derived cells. Tissue Eng Part A 14: 483-490, 2008.

52. Jeon O, Rhie JW, Kwon IK, Kim JH, Kim BS and Lee SH: In vivo bone formation following transplantation of human adipose-derived stromal cells that are not differentiated osteogenically. Tissue Eng Part A 14: 1285-1294, 2008.

53. Jurgens WJ, Oedayrajsingh-Varma MJ, Helder MN, Zandiehdoulabi B, Schouten TE, Kuik DJ, Ritt MJ and van Milligen FJ: Effect of tissue-harvesting site on yield of stem cells derived from adipose tissue: Implications for cell-based therapies. Cell Tissue Res 332: 415-426, 2008.

54. Al-Salleeh F, Beatty MW, Reinhardt RA, Petro TM and Crouch L: Human osteogenic protein-1 induces osteogenic differentiation of adipose-derived stem cells harvested from mice. Arch Oral Biol 53: 928-936, 2008.

55. Ahn HH, Kim KS, Lee JH, Lee JY, Kim BS, Lee IW, Chun HJ, Kim JH, Lee HB and Kim MS: In vivo osteogenic differentiation of human adipose-derived stem cells in an injectable in situ-forming gel scaffold. Tissue Eng Part A 15: 1821-1832, 2009.

56. Asserson DB, Orbay H and Sahar DE: Review of the pathways involved in the osteogenic differentiation of Adipose-derived stem cells. J Craniofac Surg 30: 703-708, 2019.

57. Zhang H, Kot A, Lay YE, Fierro FA, Chen H, Lane NE and Yao W: Acceleration of fracture healing by overexpression of basic fibroblast growth factor in the mesenchymal stromal cells. Stem Cells Transl Med 6: 1880-1893, 2017.

58. Deng Y, Guo T, Li J, Guo L, Gu P and Fan X: Repair of calvarial bone defect using Jaridla-knockdown bone mesenchymal stem cells in rats. Tissue Eng Part A 24: 711-718, 2018.

59. Xie Q, Wei W, Ruan J, Ding Y, Zhuang A, Bi X, Sun H, Gu P, Wang $Z$ and Fan X: Effects of miR-146a on the osteogenesis of adipose-derived mesenchymal stem cells and bone regeneration. Sci Rep 7: 42840, 2017.

60. Huang M, Qing Y, Shi Q, Cao Y and Song K: miR-342-3p elevates osteogenic differentiation of umbilical cord mesenchymal stem cells via inhibiting Sufu in vitro. Biochem Biophys Res Commun 491: 571-577, 2017.

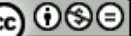

This work is licensed under a Creative Commons Attribution-NonCommercial-NoDerivatives 4.0 International (CC BY-NC-ND 4.0) License. 